\title{
Radiation Damage in Undoped CsI and CsI(Tl)
}

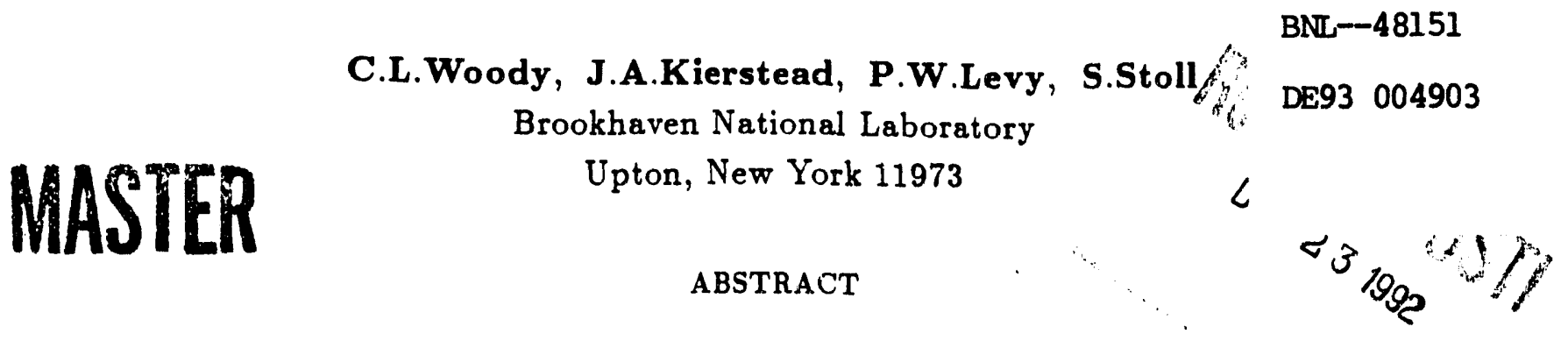

\begin{abstract}
Radiation damage has been studied in undoped CsI and CsI(TI) crystals using ${ }^{\circ 0} \mathrm{Co}$ gamma radiation for doses up to $\sim 4.2 \times 10^{\circ}$. Samples from various manufacturers were measured ranging in size from $2.54 \mathrm{~cm}$ long cylinders to a $30 \mathrm{~cm}$ long block. Measurements were made on the change in optical transmission and scintillation light output as a function of dose. Although some samples showed a small change in transmission, a significant change in light output was observed for all samples. Recovery from damage was also studied as a function of time and exposure to UV light. A short lived phosphorescence was observed in undoped CsI, similar to the phosphorescence seen in $\operatorname{CsI}(\mathrm{Tl})$.
\end{abstract}

\section{INTRODUCTION}

Studies of radiation damage in cesium iodide has generally focused on thallium doped CsI crystals due to their widespread use in particle detectors [1-5]. The data have shown that noticeable damage occurs for doses $\sim$ $10^{3}-10^{4} \mathrm{rad}$. It was assumed that the presence of thallium was responsible for the relatively high radiation sensitivity of the crystal. However, much work has recently been done on the purification of CsI raw material for the manufacture of undoped CsI crystals for fast scintillation applications. Recent results [6] have shown that undoped CsI is in fact quite radiation hard, much more so than the previous results on CsI(Tl) indicated. More recent results on $\mathrm{CsI}(\mathrm{Tl})$ have also indicated better radiation hardness than originally believed [7].

In order to study this further, a comparison has been made of radiation damage in undoped CsI and CsI(Tl). Measurements were made on the change in optical transmission and scintillation light output of a number of samples supplied by different manufacturers. Samples were exposed to ${ }^{\circ 0} \mathrm{Co}$ gamma radiation up to dose of 4.2 $x 10^{\circ}$ rad. The transmission and light output were also measured over a period of time after irradiation to study the effect of natural recovery in both materials. Recovery was also studied as a function of exposure to UV light. In addition, a short lived phosphorescence, or afterglow, was observed is ae undoped CsI which, to our knowledge, has not been previously reported. Results are given on the decay time of this phosphorescence, along with the decay time of the well known radiation induced phosphorescence in CoI(Tl).

\section{EXPERIMENTAL PROCEDURE}

The radiation measurements were carried out at two different facilities at Brookhaven National Lab. One, the Gamma Ray Radiation Facility [8], consists of a multipurpose visible and UV spectrophotometer used in conjunction with a high intensity $(\sim 20 \mathrm{KCi}){ }^{\circ 0} \mathrm{Co}$ source. This system can be used to carry out optical measurements on crystals before, during and after irradiation. Another facility, HIRDL [9], consists of a collection of ${ }^{\circ} \mathrm{Co}$ sources totaling $\sim 90 \mathrm{KCi}$ arranged in a pool of water containing long exposure tubes which provide uniform doses for samples up to $30 \mathrm{~cm}$ in length. We have used the HIRDL facility to irradiate several small samples at the same time, as well as to irradiate larger samples one at a time. The dose rate used at HIRDL was $3.4 \times 10^{4}$ $\mathrm{rad} / \mathrm{hr}$, and typically $3.8 \times 10^{4} \mathrm{rad} / \mathrm{hr}$ at the Gamma Ray Facility.

The samples used in this study were obtained frnm several sources. A number of $1^{n}$ dia. $x 1^{\text {" long cylindees }}$ were obtained from BDH [10], Horiba [11], and Quartz and Silice [12]. The HIRDL facility was used to simultaneously irradiate three samples of undoped CsI and three CsI(TI), one from each of the three suppliers, to doses of $10^{3}, 10^{4}, 6 \times 10^{4}, 2.6 \times 10^{8}, 9.0 \times 10^{8}$ and $4.2 \times 10^{6}$ rad. The transmission and light output of the samples were measured after each irradiation as described beluw. A single large $3.5 \times 3.5 \times 30 \mathrm{~cm}^{3}$ sample obtained frnm BDH was also irradiated at HIRDL and measurements were made on the change in transmission and light uutput. Two additional small samples were irradiated at the Gamma Ray Facility to measure the emission spectrum 
during irradiation and decay time of the phosphorescence after irradiation.

The initial surface conditions of the samples were not all the same even before irradiation due to the different degree of polish provided by the various manufacturers. This resulted in apparent differences in the light transmission through the samples due to surface scatter. Moreover, because CsI is a relatively soft material, and the fact that it is slightly hygroscopic, the surface conditions are easily affected by moisture or by routine handling. All samples were irradiated in a sealed container which was filled with dry nitrogen to minimize any surface deterioration due to moisture during irradiation. Before irradiation, the all samples were wiped clean with a soft, dry tissue and wrapped in aluminum foil. Unfortunately, some of the samples were inadvertently wiped with alcohol just before the first irradiation, which caused a change in the surface conditions and rendered some of the pre-irradiation transmission scans unusable. Whenever possible, the samples were also kept in total darkness to minimize their exposure to external light. Preparation of the samples for transmission and light output measurement after each irradiation was carried out under a dim red light. All measurements were typically completed within 3 hours after irradiation and the samples immediately returhed to the ${ }^{60} \mathrm{Co}$ pool to continue the exposure.

The transmission of each sample was meastired within approximately one hour after irradiation using a Hitachi U-3210 Spectrophotometer. This instrument was equipped with a large sample compartment which permitted measuring the transmission of samples up to $30 \mathrm{~cm}$ in length. It also utilized an integrating sphere to collect all refracted and scattered light from the sample, which greatly improved the accuracy of of the transmission measurement. However, due to other systematic effects, such as handling of the samples and errors in repositioning the samples inside the spectrophotometer, the accuracy of the transmission measurements was $\sim \pm 2 \%$.

The scintillation light output was measured using a Hamamatsu R2059 2" diameter photomultiplier tube with a bialkali photocathode and quarts window. The samples were wrapped in 2 layers of white reflesting teflon and placed immediately in front of the phototube with only a thin air gap ( $\$ 0.1 \mathrm{~mm}$ ) in between. No optical grease was used to couple the crystal to the phototube in order to reduce the systematic error introduced by the grease joint and to avoid having to clean the samples aiter each measurement.

The light output was measured in terms of the number of photoelectrons produced on the photocathode of the phototube per MeV of energy deposited in the crystal by a ${ }^{137} \mathrm{Co}$ gamma ray source. For the undoped CsI, the signal from the phototube was digitised using two separate LeCroy 2249W ADC's, one with a 100 ns effective gate width, used to measure the fast component, and a second with a 1 usec gate, used to measure the total light output (fast plus slow). For the CsI(TI), a single ADC with a 5 usec gate was used. The photopeak from the ${ }^{137} \mathrm{Cs}$ source was used to determine the charge output from the phototube at a given voltage for an energy deposit of $662 \mathrm{keV}$ in the crystal. The charge per photoelectron for the phototube operating at the same voltage was determined independently using an LED. Although the absolute number of photoelectrons was measured in each case, our results, for the most part, are given in terms of the relative change in light output with respect to the unirradiated sample.

The gain of the phototube, as well as other sources of systematic errors, were monitored continuously throughout the experiment using control samples of undoped CsI and CsI(Tl) which were not irradiated, but treated in every other way the same as the irradiated samples. The overall systematic error in the determination of the light output was a maximum of $\pm 5 \%$, with an $\mathrm{tms}$ of $\sim$ $2 \%$. This variation essentially determines the limit of our sensitivity to measure changes in light output due to radiation.

\section{TRANSMISSION AND LIGHT OUTPUT RESULTS}

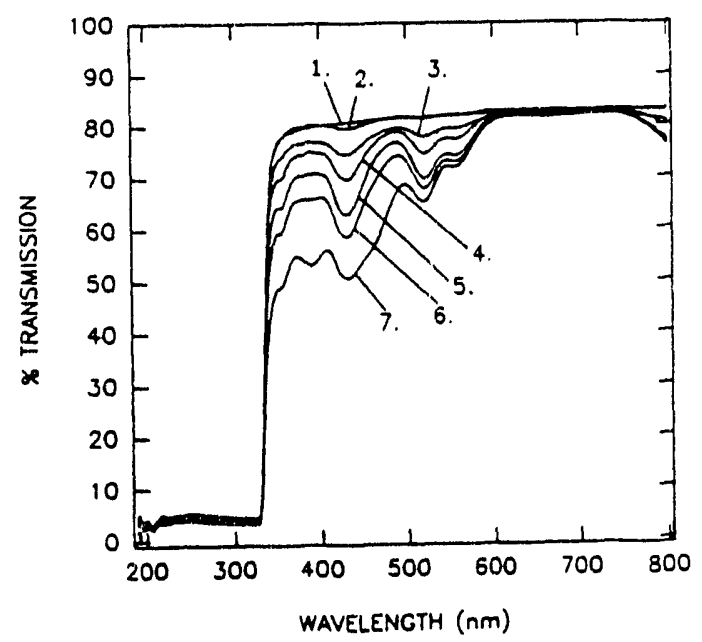

Fig. 1: Transmission vs. wavelength for the CsI(Tl) sample from BDE for doses of:(1) unirradiated, (2) $10^{3}$, (3) $10^{4}$, (4) $6 \times 10^{4}$, (5) $2.6 \times 10^{5}$, (6) $9.0 \times 10^{5}$ and (7) $4.2 \times 10^{\circ} \mathrm{rad}$.

Figure 1 shows the transmission spectrum as a function of dose for the CsI(Tl) sample from BDH. This spectrum, and all that follow, have not been corrected for the transmission loss due to surface reflection or scatter. A complex set of absorption bands are produced near the band edge, and another is seen forming near the infrared. The shift from zero transmission below the band edge is an instrumental effect due to luminescence produced in the sample by the spectrophotometer beam. The other CsI(TI) samples showed similar effects, but 

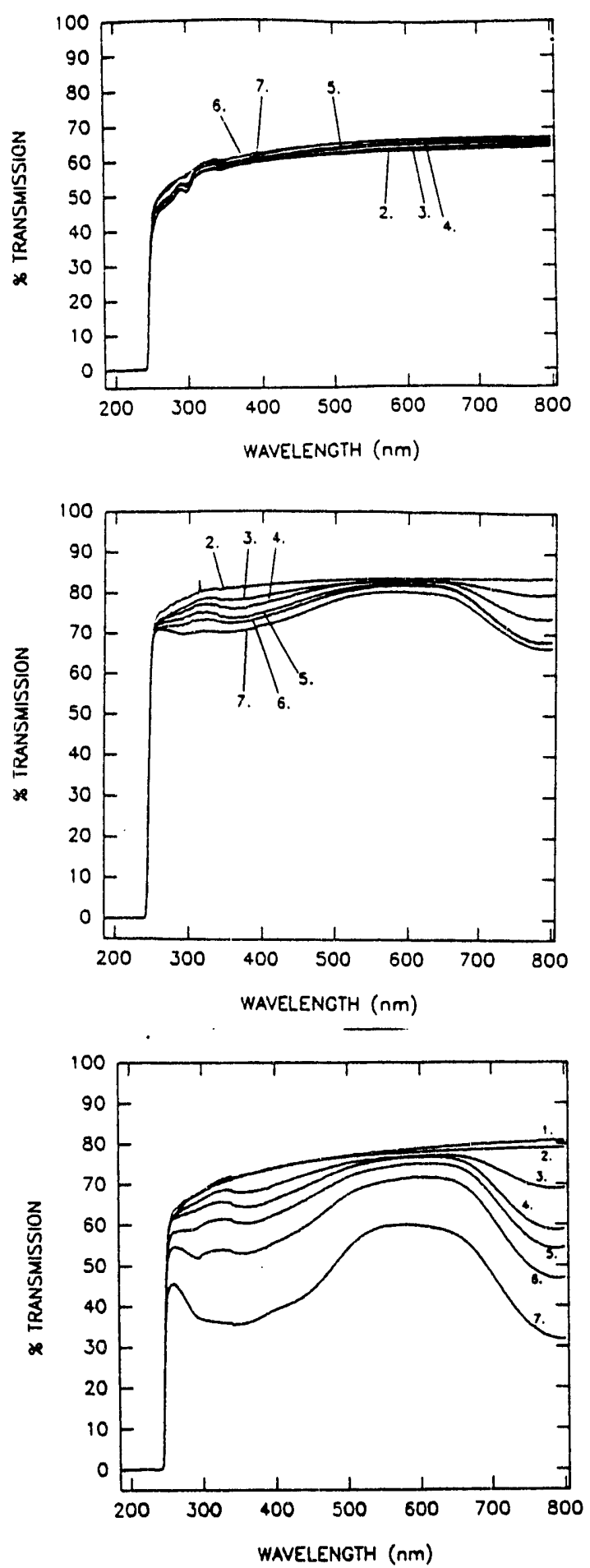

Fig. 2: Transmission vs. wavelength for 1 "dia. $x 1$ "long undoped CsI samples (a) Q\&S, (b) BDH, and (c) Horiba for doses of:(1) unirradiated, (2) $10^{3}$, (3) $10^{4}$, (4) $6 \times 10^{4}$, (5) $2.6 \times 10^{5}$, (6) $8.0 \times 10^{8}$ and $(7) 4.2 \times 10^{\circ} \mathrm{rad}$

were more affected by surfaces changes than the BDH sample, making it difficult to extract a consistent set of transmission spectra. The induced absorption is similar to that observed in ref. [1], although the amount of sbsorption is considerebly lese and the structure of the absorption bands are rather different.
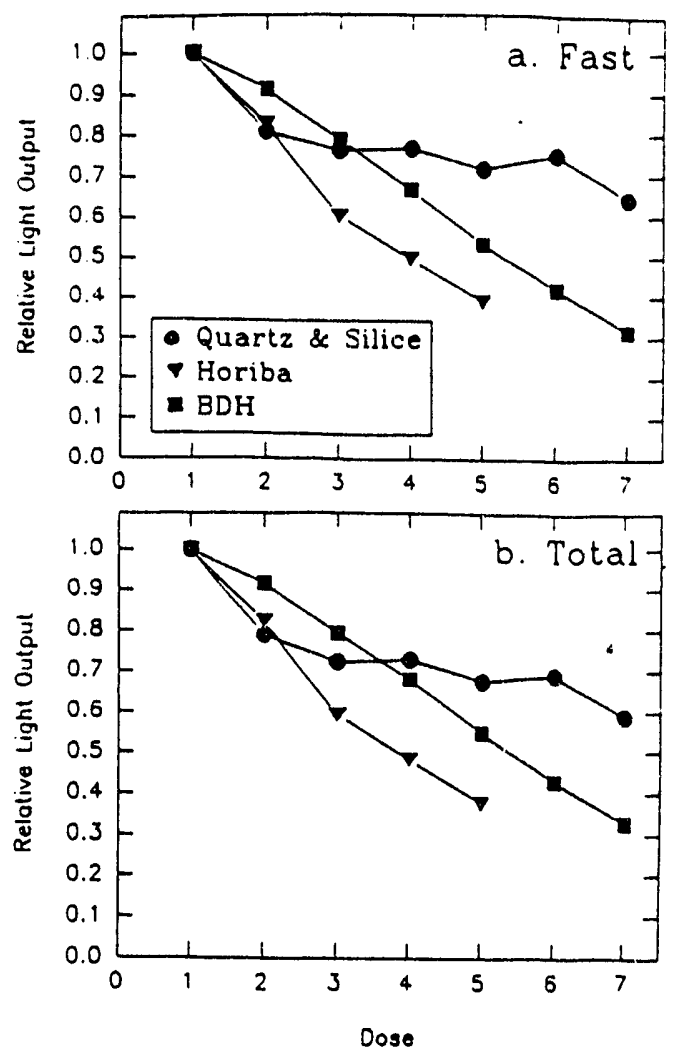

Fig. 3: Relative light output as a function of dose for fast (a) and slow (b): (1) unirradiated, (2) $10^{3}$, (3) $10^{4}$, (4) $6 \times 10^{4}$, (5) $2.6 \times 10^{5}$, (6) $8.0 \times 10^{5}$ and (7) $4.2 \times 10^{\circ} \mathrm{rad}$

Figure 2 shows the transmission spectra as a function of dose for three 1" dis. $x 1$ " long samples of undoped CsI. It is clear that different samples show varying amounts of damage. The Q\&S sample showed very little change from $10^{3}$ rad to $4.2 \times 10^{\circ}$ rad. The slight variation in transmission with increasing dose is within the systematic errors due to surface conditions. The BDH sample developed some absorption in the region of $300-400 \mathrm{~nm}$, along with a broad absorption band in the region of 800 $\mathrm{nm}$. The $800 \mathrm{~nm}$ band has been previously observed and has been tentatively identified as an F-center [13]. The Horiba crystal showed the most damage, developing more absorption in the same regions as in the BDH sample. These results are in qualitative agreement with those given in ref. [6]: This shows that the amount of damage in the material is highly sample dependent, probably caused by different levels of impurities or defects. However, the results obtained with the Q\&S sample indicate that the intrinsic radiation hardness of undoped CsI may be quite good.

Figure 3 shows the relative change in light output as a function of dose for the three undoped samples. We again see a strong sample to sample variation, with the Q\&S sample showing a 35\% loss of light output in the fast component, while the Horiba sample lost essentially all of its useful light output after a dose of $9 \times 10^{5}$ rad. This is again consistent with the type of sample to sample 

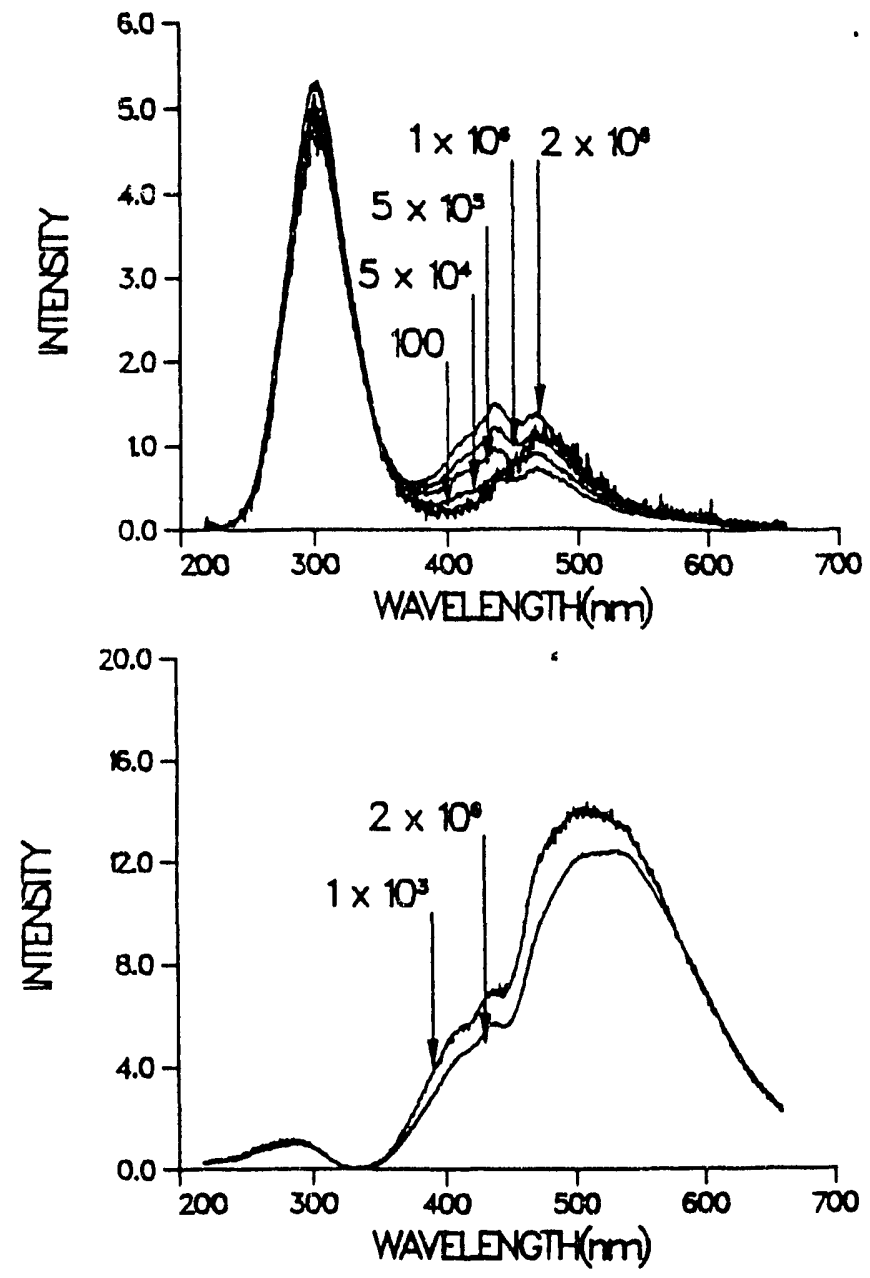

Fig. 4: Scintillation emission spectrum of undoped CsI (a) and CsI(TI) (b) measured during irradiation for various doses indicated.

variation in the light output after irradiation reported in ref. [6].

The light output of the CsI(Tl) samples could not be measured immediately after irradiation due to the strong, long lived phosphorescence. This effect is well known and has been previously reported in the literature $[1,2,3,5]$, [14]. The light output of the CsI(Tl) samples was in this case measured 40 days after the final irradiation, which was more than sufficient to allow the phosphorescence to die away. The decrease in the relative light output for the three samples was $14 \%, 24 \%$ and $48 \%$ for the Horiba, Q\&S and BDH samples, respectively. These results are rather similar to those obtained for the undoped CsI samples, and indicate better radiation hardness then previously measured [1]. This is most likely due to improvements in the quality of the pure CsI used to grow the thallium doped crystals.

The results from the light output and transmission measurements indicate an apparent loss in light output that exceeds what would be expected simply from the loss in internal transmission. This effect has also been observed in other materials [15]. In the case of the Quarts and Silice sample, there is no significant loss in transmission after the maximum dose within the measurement

error of a few percent, while the loss in light output is $30 \%$. It is clear that transmission loss alone cannot explain this effect. However, the amount of light detected by the phototube is determined by numerous factors, such as geometry, light collection efficiency, and the amount of scintillation light produced within the crystal. We have used a ray tracing simulation program [16] to study the effects of changes in the attenuation length and light collection in the crystal to see if the increased internal absorption could explain the observed loss in light output. We have found that the observed increase in absorption alone cannot explain the decrease in light output using a simple model. This conclusion was also reached in ref. [15]. We are presently using this program to try and determine if changes in surface reflectivity, or perhaps increased absorbance at the surface, could further explain this effect.

In order to determine if the amount of scintillation light produced with a crystal changes as a result of radiation, two other samples of undoped CsI and CsI(Tl) were irradiated at the Gammo Ray Facility where the scintillation emission spectrum was measured during irradiation. Figure 4 shows the results after several different doses. The specira have not been corrected for the spectral response of the spectrophotometer, and hence do not give the actual shape of the emission spectrum. Also, the arrangement of the spectrometer is not the same as that used for making the light output measurements, and the light collection efficiencies in the two setups are quite different. However, the data do give a relative comparison of the spectra for different doses. The shape of the spectrum of the undoped CsI changes significantly in the region from $400-500 \mathrm{~nm}$ from 100 to $2 \times 10^{\circ} \mathrm{rad}$. This region has been previously associated with the slow component emission [6]. However, little change occurs in the region of the fast emission (actually, a slight increase in the intensity was observed, but is presently within the systematic error of the measurement). The change in the region of the fast component certainly does not explain the decrease in light output measured after irradiation. The spectrum for the CsI(TI) shows a continuous decrease in the scintillation intensity with increasing dose, but not enough to explain the decrease in light output measured after irradiation. The second peak $\sim 290 \mathrm{~nm}$ in the emission spectrum could be due an emission from pure CsI near the surface. It is possible that radiation effects near the surface, either in the emission or absorption, could cause changes in the light collection efficiency which could give rise to the observed loss in scintillation light output after irradiation. However, it may also be possible that the apparent scintillation efficiency during irradiation may be different than after irradiation due to the large amount of ionization occurring in the crystal during irradiation. We are presently exploring both of these possibilities with further measurements and calculations using the simulation program to try and fully understand these effects. 


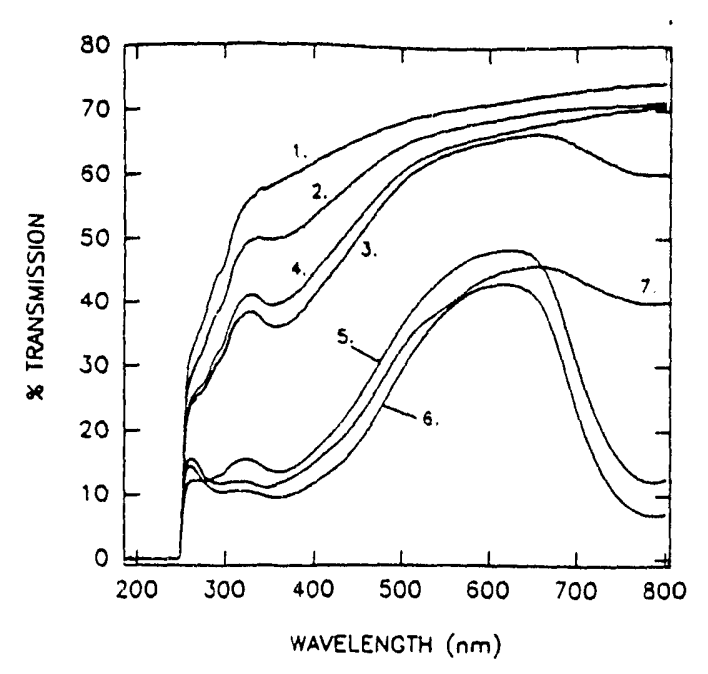

Fig. 5: Transmission spectrum for a $3.5 \times 3.5$ $\times 30 \mathrm{~cm}^{3}$ undoped CsI sample from BDH. Curves indicate (1) unirradiated, (2) $10^{3} \mathrm{rad},(3) 10^{4} \mathrm{rad}$, (4) 22 hrs. after $10^{4} \mathrm{rad}$, (5) $10^{5} \mathrm{rad},(6) 10^{6} \mathrm{rad}$, (7) 24 hrs after $10^{\circ}$ rad.
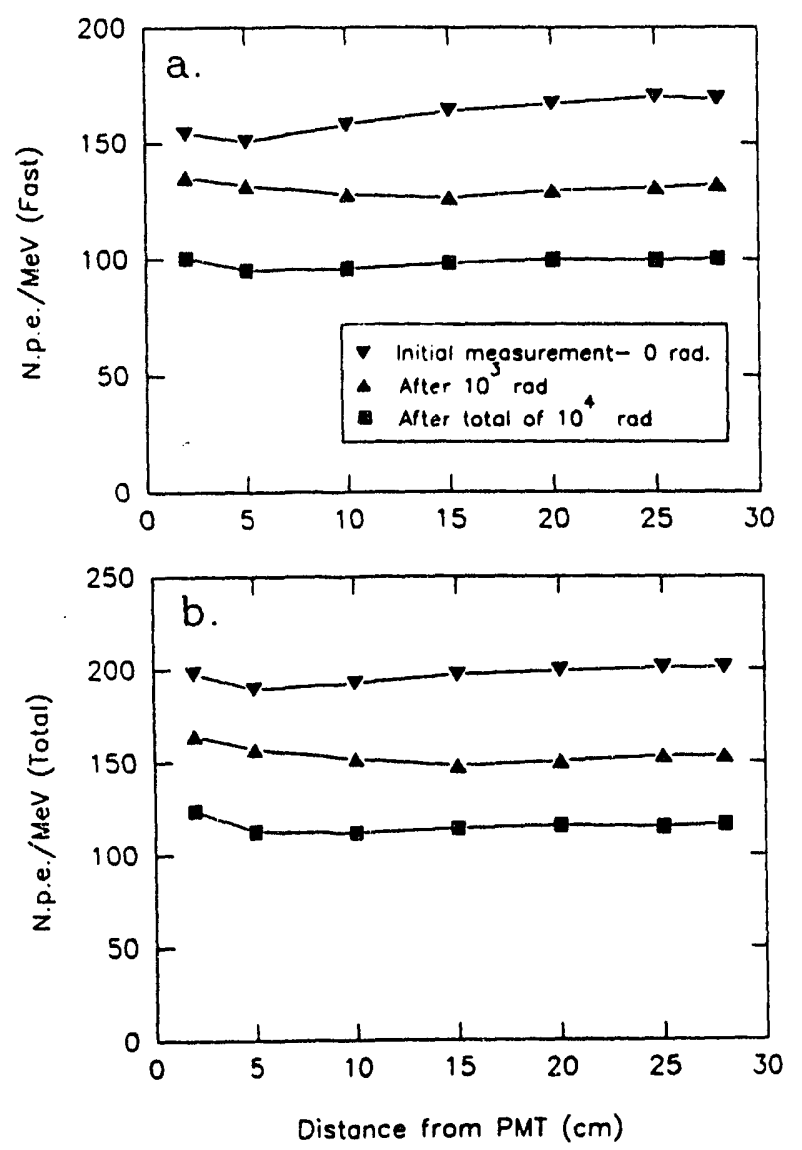

Fig. 6: Light output for fast (a) and total (b) as a function of position along the length of $3.5 x$ $3.5 \times 30 \mathrm{~cm}^{3}$ undoped CsI crystal from BDH after $10^{3}$ and $10^{4} \mathrm{rad}$.

The large $3.5 \times 3.5 \times 30 \mathrm{~cm}^{3}$ undoped CsI crystal supplied by BDH was also irradiated at the HIRDL facility. The sample was irradiated in a sealed container of dry nitrogen in the same manner as the small samples. Figure 5 shows the transmission along the longitudinal axis for doses from $10^{3}$ to $10^{6}$ rad. A substantial amount of induced absorption is observed. Figure 6 shows the light output as a function of position along the sample after $10^{3}$ and $10^{4} \mathrm{rad}$. The light output was too low to measure at all positions along the crystal for higher doses. The loss in light output at lower doses appears to be rather uniform along the length. However, a measurement made eight days after the irradiation showed that the fast component light output had recovered to $\sim 23 \%$ of its original value near the end closest to the phototube, although, the light output was still too low to measure near the middle and far end of the crystal. This indicates that there was a position dependence to the damage at higher doses, implying that there may have been a higher concentration of impurities at one end.

\section{RECOVERY}

We have studied the natural recovery of both the transmission and light output of several samples with time, and with exposure to UV light from a strong mercury lamp. Figure 7 shows the recovery in the light output for the Q\&S and BDH samples. A small amount $(\sim 10 \%)$ of recovery with time is observed in both the fast component and total light output. However, no additional recovery is induced by exposure to UV light.
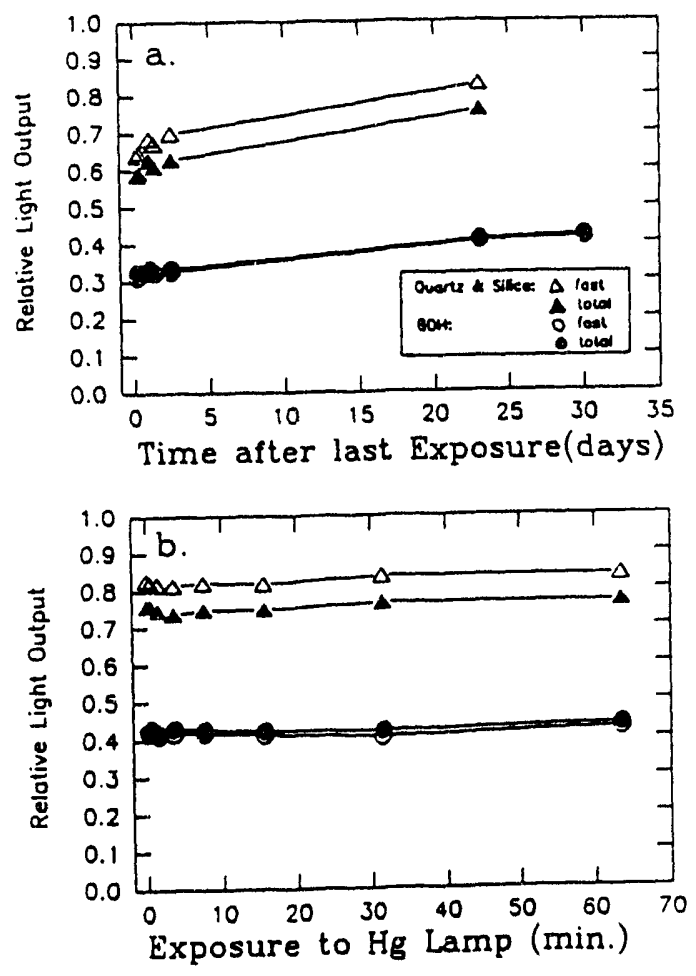

Fig. 7: Recovery in relative light output for Q\&S and BDH samples (a) as a function of time, and (b) as a function of exposure to $U V$ light. 

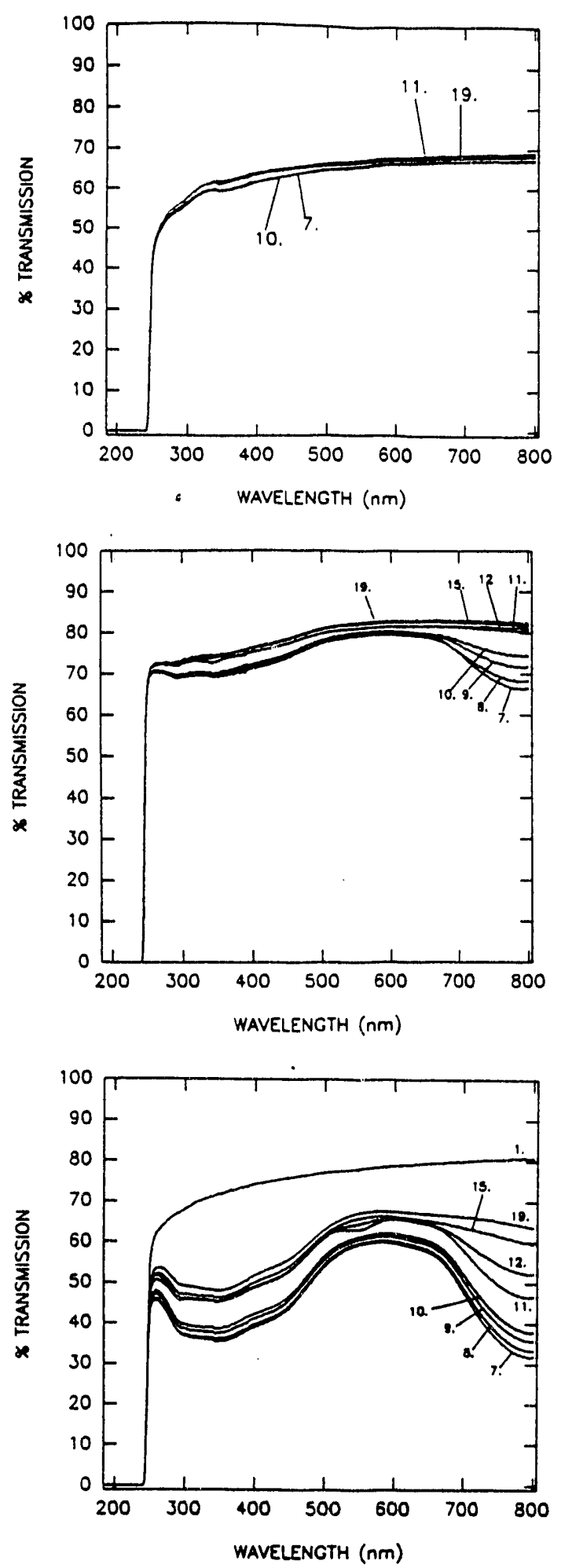

Fig. 8: Recovery in transmission of undoped CsI samples (a) Q\&S, (b) BDH, and (c) Horiba: (1) unirradiated (7) $1 \mathrm{hr}$. after $4.2 \times 10^{\circ} \mathrm{rad},(8)$ after $T$ hrs., (8) after 30 hrs., (10) after 54 hrs., (11) after 20 days (12) after 26 days, (15) after 26 days +3.5 minute exposure to UV light, (10) after 28 days + 63.5 minute exposure to UV light.

Figure 8 shows the change in transmission in the undoped samples measured at various times after the final

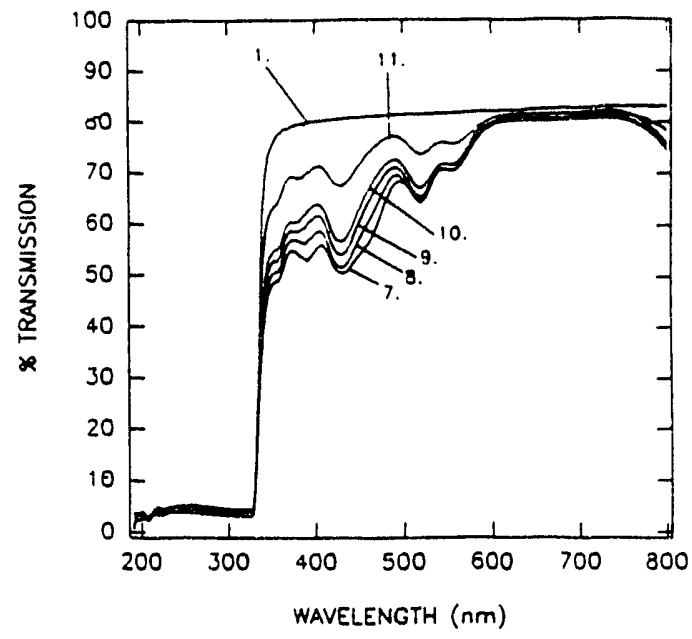

Fig. 8: Recovery in transmission of $\mathrm{CsI}(\mathrm{Tl})$ sample: (1) unirradiated (7) $1 \mathrm{hr}$. after $4.2 \times 10^{\circ}$ rad, (8) after 5 hrs., (8) after 28 hrs., (10) after 56 hrs., (11) after 22 days

irradiation, and after several exposures to the mercury lamp. Some recovery is seen in the $300-400 \mathrm{~nm}$ region for the BDH and Horiba samples, although more recovery occurs in the region of the F-center band, particularly for the BDH sample.

Figure 9 shows the recovery with time in the transmission of the BDH CsI(Tl) sample. Significant recovery is seen in the region around the band edge.

\section{PHOSPHORESCENCE}

Both the undoped and thallium doped CsI crystals exhibit phosphorescence after irradiation. Particularly in the thallium doped crystals, this phosphorescence is sufficiently intense to interfere with or prevent measuring the scintillation light output after irradiation. The phosphorescence decay of both types of samples is show $n$ in Figure 10. Both curves are normalized to the same point at sero time. However, the phosphorescence from the doped sample was roughly 100 times stronger than from the undoped. The curve for the doped sample shows a plateau for the first few seconds after irradiation which was due to an instrumental saturation effect, and this part of the curve was not used in the subsequent fits to the decay times. The decay curves have been resolved into several exponential components. While two components fit the curves quite well, better fits were obtained when they were resolved into three components. The numerical values obtained from the fits, giving the $(1 ; e)$ decay times and fraction of the total intensity for each component, are given in Table $I$. 


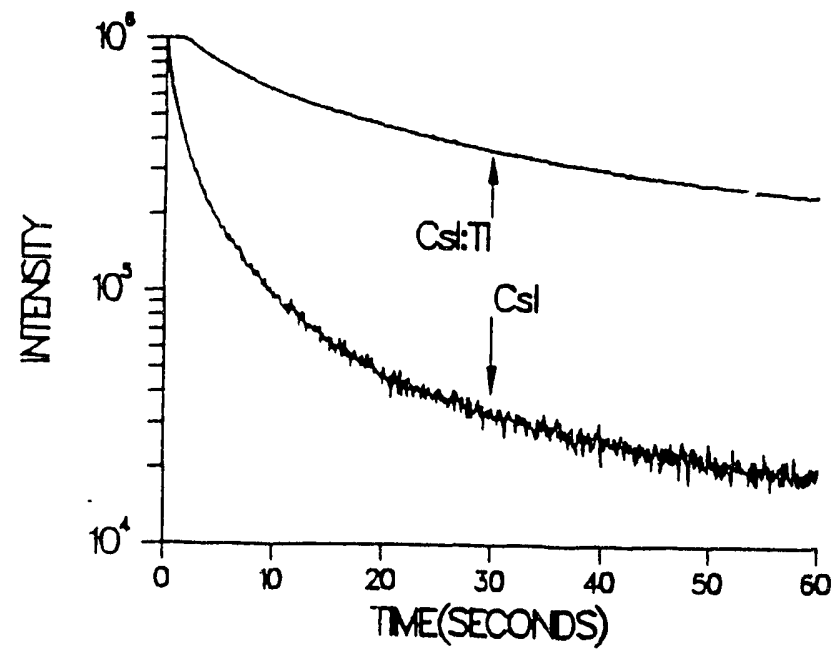

Fig. 10: Phosphorescence decay curves for undoped CsI and CsI(TI)

Table I

Components of Phosphorescence Decay in Undoped CsI and CsI(Tl)

\begin{tabular}{|c|c|c|c|}
\hline Sample & \multicolumn{3}{|c|}{ Components $(\tau$ in seconds, ()$=\%)$} \\
\hline & $\tau_{1}$ & $\tau_{2}$ & $\tau_{3}$ \\
\hline CsI & $0.7(7)$ & $4.0(34)$ & $39(59)$ \\
$\mathrm{CsI}(\mathrm{Tl})$ & $4.1(3)$ & $14.7(12)$ & $111.6(85)$ \\
\hline
\end{tabular}

It was noted that some residual phosphorescence was observed in undoped CsI which persisted for several days. No attempt was made to measure this decay time, but at least one additional decay component with a much longer decay time must be present.

The emission spectrum of the phosphorescence from the thallium doped sample was also measured, but with a poor signal to noise ratio. It extends from roughly $300 \mathrm{~nm}$ to $700 \mathrm{~nm}$ and contains at least two, and possibly more, components. The emission spectrum from the undoped Csl was too weak to measure with our presen s apparatus.

\section{CONCLUSIONS}

Radiation damage has been measured in undoped CsI and CsI(Tl) and both show rather similar effects. Absorption bands are produced in both materials, the location and intensity of which are highly sample dependent. One undoped sample showed a minimal amount of induced absorption, indicating that the intrinsic radiation resistance of undoped CsI may be rather high. However, all samples showed a decrease in scintillation light output after irradiation, which was greater than can be explained by a simple loss in light due to absorption. A Monte Carlo calculation including the effects of multiple reflections shows that the increased absorption due to radiation cannot easily explain the observed loss in light. Measurements of the emission spectra of both undoped CsI and CsI(Tl) indicate only a small change in the scintillation intensity during irradiation. This implies that the loss in detected light is due to some other effect, such as a change in the light collection efficiency, or that the scintillation intensity measured during irradiation is not the same as after irradiation. Additional measurements and calculations are currently under way to try to better understand these results.

A small amount of natural recovery was observed in samples kept in the dark at room temperature for a period of roughly 30 days. Very little recovery was observed with exposure to UV light. A short lived phosphorescence was observed in undoped CsI, along with a weaker component with a much longer decay time. This is similar to the type of phosphorescence which has been observed in CsI(TI).

Based on the limited number of samples measured, the large sample to sample variations indicate probable differences in the purity of the material from different manufacturers. If the observed radiation damage is related to impurities or defects, it is likely that the radiation hardness of both undoped and doped CsI can be enhanced by improved growing techniques. However, much additional work must be done to understand the possible causes of the radiation damage before significant improvements can be made. 


\section{ACKNOWLEDGMENT}

This work was supported in part under Department of Energy Contract No. DE-AC02-CH7600016.

\section{REFERENCES}

1. M.Kobayashi and S.Sakuragi, Nucl. Inst. Meth. A254 (1987) 275 .

2. Ch.Bieler et.al., Nucl. Inst. Meth. A234 (1985) 435.

3. H.Grassmann et.al., Nucl. Inst. Meth. 228 (1985) 323.

4. S.Schlogl et.al., Nucl. Inst. Meth. A242 (1985) 89.

5. CLEO II Updated Proposal, CLNS 85/634 (1985) p.204.

6. C.Woody et.al., IEEE Trans. Nucl. Sci.Vol. 37 (1990) 492..

7. D.Renker, Proceedings of the International Workshop on Instrumentation for High Luminosity Hadron Colliders, Barcelona, Spain, Sept. 1989, CERN 89-10, Vol.2.

8. P.W.Levy, J. Phys. Chem. Solids, Vol. 52, No. 1 (1991) 319.

9. The High Intensity Radiation Development Laboratory (HIRDL) at BNL.

10. BDH Chemical Co. Ltd., now CRYSTRAN Merck Ltd., Poole, England.

11. Horiba Crystal Products, a division of Horiba Instruments, Inc., Kyoto, Japan.

12. Quartz and Silice Company, a division of Saint Gobain, Nemours-Cedex, France.

13. P.Avakian and A.Smakula, Phys. Rev. 120 (1960) 2007;

C.Bates, A.Salau and D.Leniart, Phys. Rev. B15 (1977) 5963.

14. M.R.Farukhi, IEEE.Trans. Nucl. Sci. NS-29 (1982) 1237.

15. See other contribution to this symposium, Radiation Effects in $\mathrm{BaF}_{2}$ Crystals, C.Woody et.al., and A.Murakami et.al., Nucl. Inst. Meth. A301 (1991) 435.

16. We are grateful to L.Littenberg from BNL for proDISCLAIMER viding us with the ray tracing program.

This report was prepared as an account of work sponsored by an agency of the United States Government. Neither the United States Government nor any agency thereof, nor any of their employees, makes any warranty, express or implied, or assumes any legal liability or responsibility for the accuracy, completeness, or usefulness of any information, apparatus, product, or process disclosed, or represents that its use would not infringe privately owned rights. Reference herein to any specific commercial product, process, or service by trade name, trademark, manufacturei, or otherwise does not necessarily constitute or imply its endorsement, recommendation, or favoring by the United States Government or any agency thereof. The views and opinions of authors expressed herein do not necessarily state or reflect those of the United States Government or any agency thereof. 

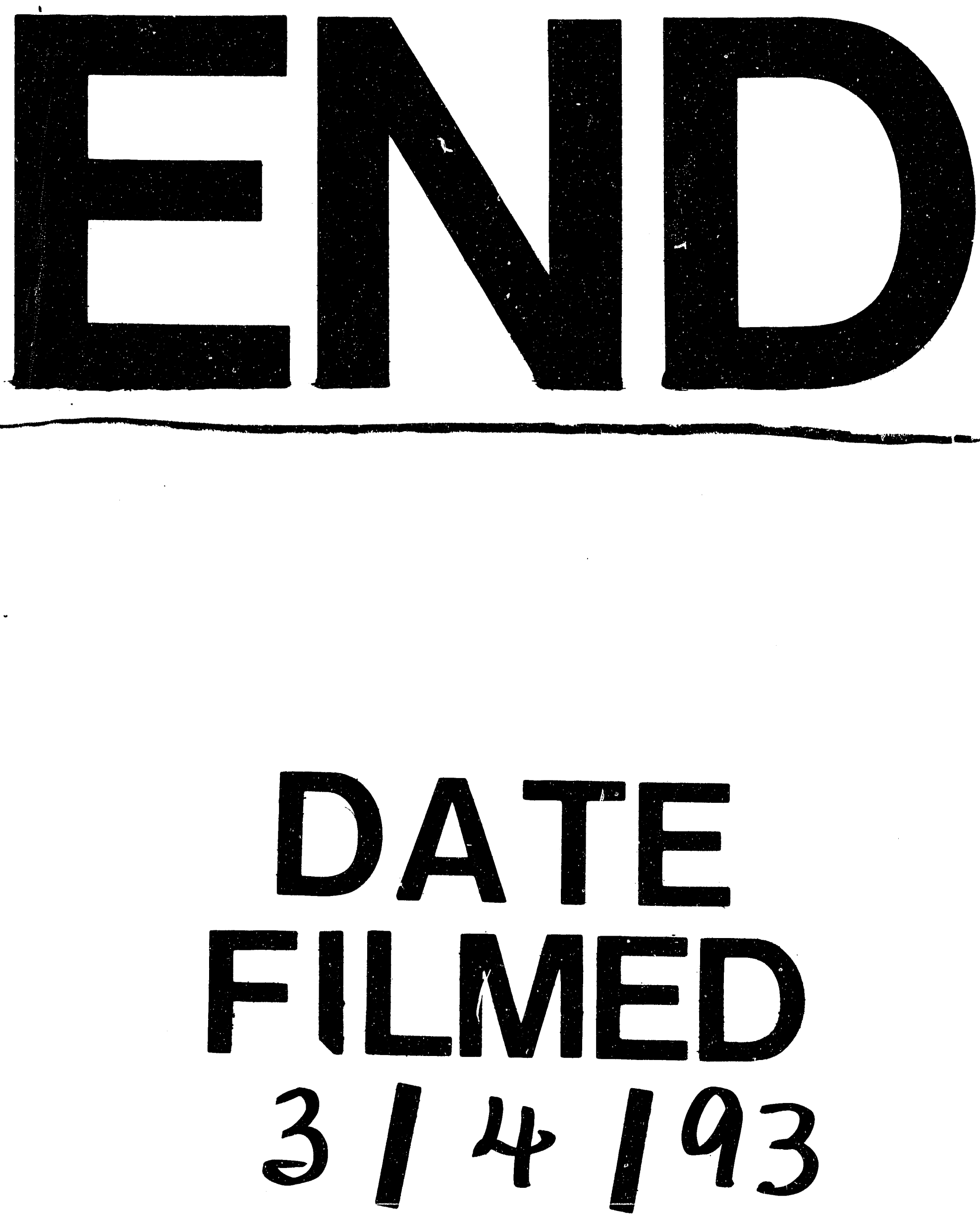


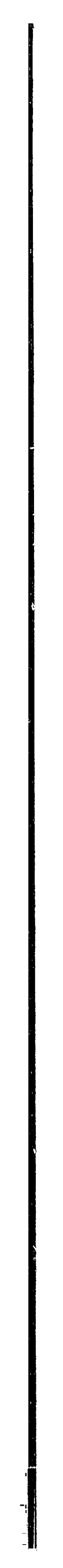

